\title{
Aktive Mitarbeit an der Umgestaltung der Wirtschaft
}

\section{Das Verhältnis zwischen Unfernehmen und Umweltverbänden ist traditionell eher konfliktbestimmt. Doch die extreme Polarisierung der 70er Jahre zwischen Umweltschützern und der Industrie ist nicht mehr zeitgemäß.}

$\mathrm{H}$ Von Jutta Baßfeld

eute wird in vielen Unternehmen aus den unterschiedlichen Interessenlagen heraus stark über Ökologie gestritten. Daher ergänzen seit über fünf Jahren Wirtschaftskooperationen das traditionelle umweltpolitische Instrumentarium des BUND und tragen der Entwicklung Rechnung, daß viele Unternehmen sich in ökologischer Hinsicht engagieren. Dadurch eröffnet sich die Möglichkeit, den ökologischen Strukturwandel aktiv voranzutreiben.

In der projektbezogenen Zusammenarbeit mit Unternehmen sieht der BUND eine Möglichkeit, einen lösungsorientierten Beitrag zur Ökologisierung der Gesellschaft zu leisten. Ohne das Mitwirken der Wirtschaft ist ein grundlegender ökologischer Wandel nicht zu erreichen.
Kooperation bietet die Chance, den öko-sozialen Visionen in der Realität ein wenig näher zu kommen.

\section{Wirkung nach innen und außen}

Kooperationen wirken in zweifacher Hinsicht. Unmittelbar in den Unternehmen führen sie zu ökologischen Verbesserungen und stoßen darüber hinaus ökologische Veränderungen in Märkten und im politischen Umfeld an. So können in Unternehmen mit Hilfe des Umweltwissens des BUND ökologisch fundierte Maßnahmen ergriffen werden. Außerdem löst eine Kooperation in Unternehmen erhebliche Motivations- und Mobilisierungseffekte aus, die eine Umsetzung der ökologischen Maßnahmen erleichtert. Die Zusammenarbeit mit dem BUND wirkt über die Grenzen der eigentlichen
Kooperation hinaus: Sie erleichtert es Unternehmen, ökologische Forderungen oder neue ökologische Standards am Markt durchzusetzen. Durch strategische Allianzen von Unternehmen und BUND können zudem politische Forderungen verstärkt werden. Dies zeigte die Öko-Steuer-Initiative des BUND, in der sich 16 namhafte Unternehmen öffentlich in einer bundesweit geschalteten Anzeige für die Einführung einer Öko-Steuer aussprachen.

\section{Nutzen aus der Zusammenarbeit}

Für beide Partner ergeben sich vielfältige Nutzen aus der Zusammenarbeit. Unternehmen profitieren vom ökologischen Wissen des BUND und seiner Glaubwürdigkeit, von seiner Fähigkeit, Umweltsachverhalte verständlich $\mathrm{zu}$ machen und von der Unterstïtzung in der Öffentlichkeit bei ökologischen Neuerungen.

Für den BUND eröffnen Wirtschaftskooperationen in mehrfacher Hinsicht neue umweltpolitische Gestaltungsoptionen. Durch die Veränderung von Produktions-, Produkt- und Handelspolitik der Unternehmen werden un-mittelbare Umweltentlastungseffekte erzielt. So konnten im Laufe der Kooperation mit dem Kauf- und Warenhaus HERTIE zahlreiche ökologisch problematische Produkte aus dem Sortiment eliminiert und Hersteller zur Umstellung ihrer Rezepturen bewegt werden. Ferner unterstiitzt der BUND

\section{Kooperationsprojekte beim BUND}

\section{HERTIE Kouf- und Warenhaus GmbH}

Die Kooperation besteht bereits seit 5 Jahren. Wichtige Bousteine der Zusammenarbeit sind die Unterstützung des organisationsinternen Ökologisierungsprozesses und der Sortimentscheck, bestehend aus drei Grundelementen: Das bestehende Sorliment wird nach ökologischen Kriterien überprüft, umstrukiuriert und für das veränderte Sortiment eine gemeinsame Verbroucherinformation entwickelt.

\section{Unternehmensinitiative}

\section{für eine ökologische Steverreform}

16 namhafte Unternehmen (AEG, Auro, Berkel, Body Shop, Werner \& Mertz, 0770 usw.) setzten sich 1994 gemeinsam mit dem BUND für eine ökologische Steverreform ein. Es wurde eine gemeinsame Erklärung "Umsteuern in der Krise" verfaft und eine bundesweite Anzeigenkampagne "Die große Kodition ist da!" durchgeführt.

\section{Deutsche Angestellten-Krankenkasse (DAK)}

Zu verkehrspolitischen Themen (Ozon, Lärm etc.) fanden zahlreiche lokale Kooperationen zwischen BUND- gruppen und DAK-Geschäftsstellen statt. Für die Akrionen wurden Aktionstips, Broschüren u.ö. zur Verfügung gestellt.

\section{Tupperware}

Im Rahmen der Kampagnge zur Abfallvermeidung wurde 1994 unter dem Titel "Bitte ohne Drumherum" für den verpackungsarmen Einkauf mit mitgebrachten Mehrwegbehältern geworben. BUND-Gruppen motivier: ten die Lebensmittelgeschäfte vor Ort zum Mitmachen. Die Tupperberaterinnen warben bei den Verkautsparties für den verpackungsarmen Einkaut. Die Geschäfte, in denen inzwischen verpackungsarm eingekautt werden kann, wurden in einem Einkoufsführer zusammengefaßt.

\section{Alpirsbacher Klosterbräu}

1994 wurde ein Standardbierkasten entwickelt, um gegen die zunehmende Individualisierung von Bierküsten und die daraus resultierende Gefährdung von Mehrwegsystemen vorzugehen. Durch den Standardkasten können bei einem ausreichend großen Pool Leergutfahrten und Sortieroufwand eingespart werden.

\section{neuform-Reformhäuser}

1995 wurden Kino-Spots für den ökologischen Landbau in über 100 Kinos plaziert. Parallel fanden rund um und in den Kinos Aktionen der örtlichen BUNDgruppe und des Reformhauses statt. 1996 wurde die Kooperation mit einer Kampagne zur Autklöinng der Verbroucher über die Risiken gentechnisch veränderter Lebensmittel fortgesetzt. In ca. 50 Städten fanden lokale Aktionen mit einer cufblasbaren Riesentomate statt.

\section{Deutsche Bahn AC}

1995 wurde ein Gutachten zur umwelffreundlichen Gestaltung des mobilen Servicebereiches erarbeitet und oufgezeigt, in welchen Bereichen Dosen und Einwegmüll durch Mehrwegsysteme ersetzi werden können. 1996 wurde die Praktikabilität der Mehrwegalternativen getestet.

\section{Cherry Mikroschalter GmbH}

Um einen Einstieg in die Ökologisierung der Computerbranche zu schaffen, wurde 1996 ein Kritierienkotalog für eine ökologisch optimierte Tastatur entwickelt. Die erste Serie der Öko-Tastatur wird im Herbst 1997 auf dem Markt sein. 
besonders umwelffreundliche Firmen, Produkte und Dienstleistungen, um durch diese Pionierleistungen zur Nachahmung in der Branche anzuregen. So erfahren z.B. alternative Hersteller über die Einlistung ihrer ökologisch fortschrittlichen Produkte bei HERTIE eine Stärkung ihrer Marktposition. Und auch neue Zielgruppen können über die BUNDpolitik informiert werden. So warben z.B. Tupper-Beraterinnen während der Aktion „Bitte ohne Drumherum“ für den verpackungsarmen Einkauf. Die Verbraucher wurden unmittelbar mit der BUNDpolitik konfrontiert und erhielten gleichzeitig Anregungen für umweltentlastende Verhaltensweisen.

\section{- Kooperationskriterien}

Das Thema Wirtschaftskooperationen ist beim BUND seit jeher umstritten. Der Bereich wurde 1992 erstmalig in die programmatische Linie des BUND aufgenommen. Nach einer „Probezeit" von drei Jahren beurteilte eine deutliche Mehrheit des Verbandes die Ergebnisse der Kooperationsprojekte positiv und entschied sich für eine Fortsetzung dieser Aktivitäten. Nach wie vor schätzen jedoch Teile des BUND die Risiken, die Wirtschaftskooperationen für den Verband mit sich bringen könnten (z.B. der Verlust an Glaubwürdigkeit), relativ hoch ein und stehen dem Instrument skeptisch gegenüber. Aus diesem Grunde werden bereits im Vorfeld der Entscheidung über eine Kooperation durch den Bundesvorstand Stellungnahmen der relevanten Gremien eingeholt. Ferner wurden 1995 die Kriterien zum Umgang mit Wirtschaftskooperationen teilweise neu definiert:

- Die Zusammenarbeit mit Unternehmen ist grundsätzlich projektbezogen und an konkrete Aktionen gebunden.

- Mit einigen Unternehmen sind Kooperationen grundsätzlich ausgeschlossen (z.B. Unternehmen der Rüstungsindustrie, der Atomindustrie, der Energiewirtschaft im Hinblick auf reinen Stromverkauf, der Chemieindustrie, der Automobilindustrie), da sie von ihrer Unternehmensaufgabe im krassen Gegensatz zur BUNDpolitik stehen.

- Öko-Sponsoring ist grundsätzlich verboten.

\section{- Öko-Sponsoring contra Wirtschaftskooperationen}

Der BUND lehnt Öko-Sponsoring grundsätzlich ab. Durch ein pauschales Entgelt der Umweltaktivitäten eines Unternehmens nach dem Muster Geld gegen Logo (z.B. für Produktwerbung) befürchtet der BUND einen Verlust seiner wirtschaftlichen Unabhängigkeit und seiner Glaubwürdigkeit. Kooperationen mit Partnern aus der Wirtschaft sind beim BUND immer projektbezogen und werden nur dann angestrebt, wenn sie auf einer konkreten inhaltlichen Ebene stattfin- den. Zielsetzung aller Kooperationen ist die ökologische Umgestaltung der Wirtschaft entsprechend dem umweltpolitischen Programm des BUND. So wurde z.B. gemeinsam mit den Reformhäusern eine Aufklärungskampagne über die Risiken gentechnisch veränderter Nahrungsmittel initiiert.

Durch die starke Projektbezogenheit ist eine pauschale Vereinnahmung des BUND-Images durch die Kooperationspartner nicht möglich - eine reine Fassadenbegrünung ist ausgeschlossen. Nur bei Erreichung eines ökologischen Erfolges in dem jeweiligen Projekt ist nach Abstimmung mit dem BUND ein gemeinsamer öffentlicher Auftritt z.B. am Point of Sale oder in der Presse- und Öffentlichkeitsarbeit möglich. Auf diese Art und Weise werden bei einer längerfristigen Zusammen-arbeit die einzelnen Projekte in einem Aushandlungsprozeß immer wieder neu konzipiert, um eine ständige Hinterfragung des Anspruchsniveaus der Zusammenarbeit sicherzustellen.

\section{Die Autorin}

Julta Babfeld ist Referentin für Wirtschafiskooperationen beim BUND in Bonn.

Kontakt: BUND, Bundesgeschäfisstelle, Im Rhein garten 7, 53225 Bonn, Tel. (0228) 40097-0, Fox 4009740

\section{Literaturliste zum Thema Kooperation und Umweltschutz}

A. Aulinger: (Ko-)Operation Ökologie. Kooperationen im Rahmen ökologischer Unternehmenspolitik. Marburg 1996 H.L. Batt: Kooperative regionale Industriepolitik. Frankfurt/M. 1994 (=Beiträge zur Politikwissenschaft 57) V. Belzer: Unternehmenskooperationen, Effolgsstrategien und Risiken im industriellen Strukturwandel. München 1993

M. Birke, B. Hurrle, G. Hurrle U. V: Mertens ( $\mathrm{Hg}$.): Unternehmenskooperation und gewerkschaftliche Interessensvertretung. München 1995 M. Brockhous: Gesellschaftsorientierte Kooperation im ökologischen Kontext: Perspektiven für dynamisches Umweltmonogement. Wiesboden 1996

P. Cooke: Innovative regional dusters: The automotive and electronics industries in Wales. Cordiff 1995 (=Regional Industrial Research Report 19)

R. Danielzyk, J. ORenbrügge: Lokale Handlungsspielrüume zur Gestaltung internationalisierter Wirtschaftsräume. In: Zeisschrift für Wirtschaftsgeographie, H.1.2, 1996, S. 101.112

Friedrichsdorfer Büro für Bildungsplanung ( $\mathrm{Hg}$.): Lernende Region. Kooperationen zur Verbindung von Bildung und Beschäftigung in Europo. Salzgitter, Berlin 1994 B. Flieger et al. (Hg.): Gemeinsam mehr erreichen.
Kooperation und Vernetzung alternativ-ökonomischer Betriebe und Projekte.

D. Fürst: Regionalkonferenzen zwischen offenen Netzwerken und fester Institutionalisierung. Getsenkirchen 1995 (=Institut für Arbeit und Technik)

M. Glagow, H. Willke (Hg.): Dezentrale Gesellschaftssteuerung. Probleme der Integration polyzentrischer Gesell. schaft. Pfoffenweiler 1987

G. Grabher: The embedded firm. London, New York 1993 H. Kilper: Steverungseffekte und Legitimation regionaler Netzwerke. Gelsenkirchen 1995 (=Institut für Arbeit und Technik)

J. Knieling: Intermediäre Organisationen und kooperative Regionalentwicklung. In: RUR 2, 1994, S. 116. 126

W. Krumbein $(\mathrm{Hg})$ : Ökonomische und politische Netzwerke in der Region. Beiträge ous der internatio nalen Debatte. Münster, Hamburg 1994 (=Politik und Ökono: mie 1)

K. Lompe, A. Blöcker, B. Lux, U. O. Syring: Regionalisierung als Innovationsstrategie. Die VW-Region auf dem Weg von der Automobil- zur Verkehrskompetenzregion. Berlin 1996

B. Marin, R. Mayntz (Hg.): Policy Networks. Empirical
Evidence and Theoretical Considerations. Frankfurt/M., Boulder/Colorado 1991

OIKOS (Hg.): Kooperationen for die Umwelt. Im Dialog zum Handeln. Zürich 1994

D. Rehfeld: Vernetzte Ökonomie und regionale Folgen. Dichte. Werkstattbericht TU München, Fakultait für Archi tektur. München 1995, S. 47.57

U. Schneidewind: Ökologisch orientierte Kooperation ous betriebswirtschaftlicher Sicht. In: Unweltwirtschattsforum 4/1995

J. Simmie: Innovation, Nefworks, and Learning Regions? London 1996 (=Regional Policy and Development 18)

H. Spehl: Nachhalitige Regionalentwicklung - ein never Ansaiz für das Europa der Regionen. In: B. Gahlen, H. Hesse, H.H. Ramser (Hg): Standort und Region - Neue Ansähze zur Regionaläkonamik. Tübingen 1995, S. 307.330

J. Sydow et.al: Organisation von Netzwerken. Opladen 1995

H. Weidner: Umwelikooperation und alternative Konfliktregelungsverfahren in Deutschland. Zur Entstehung eines neuen Politiknetzwerkes. WZB-paper $96-302$ 
(c) 20I0 Authors; licensee IÖW and oekom verlag. This is an article distributed under the terms of the Creative Commons Attribution Non-Commercial No Derivates License (http://creativecommons.org/licenses/by-nc-nd/3.o/), which permits unrestricted use, distribution, and reproduction in any medium, provided the original work is properly cited. 